announced that it will be revising the 1992 edition of the "Guidelines for Construction and Equipment of Hospital and Medical Facilities," which contain the minimum program, space, equipment, and engineering design requirements for medical facilities, including specifications for isolation rooms and handwashing facilities.

More than 46 states use either the current or earlier editions of the guidelines for public health or licensure programs. Beginning in 1995, the Joint Commission on Accreditation of Healthcare Organizations is proposing the adoption of the 1992-1993 edition in its standards for all newly constructed, renovated, or modernized healthcare facilities seeking accreditation.

The AIA invites all interested parties to submit proposed revisions to update the existing guidelines to the AIA by June 17, 1994. To obtain the special form for proposed revisions, telephone Todd Phillips of the AIA at (202) 6267366 or FAX (202) 6267518. A copy of the guidelines can be obtained from AIA (800) 3652724.

\section{Gloves Found to Reduce Volume of Blood Transferred During Needlestick Injuries}

Gloves were found to reduce the transferred blood volume during simulated needlestick injuries by $46 \%$ to $86 \%$. These findings were reported by Dr. Mast et al from the University of California, San Francisco, in a study that evaluated the factors that affected blood volumes transferred during needlestick injuries. The study was conducted with two simulated percutaneous needlestick injury models to evaluate predictors of blood volume transfer. These included an in vitro model that passed prepared needles through prefiltered paper and an ex vivo model that used porcine skin.

Needle size and depth of needle penetration were found to be significantly associated with the volume of blood transferred by hollow-bore and suture needles. The volume of blood transmitted increased as the depth increased and larger blood volumes were transferred with larger needles. The volume of blood transferred was within the same order of magnitude for all resting conditions. Glove material of any type (eg, vinyl or latex) was associated with a reduction in blood volume transferred by both hollow-bore and suture needles. The mean percentages of reduction in blood volume transfer when single latex gloves were used during hollow-needle simulations were $46 \%$ in the in vitro model and $63 \%$ in the ex vivo model. For suture needles in both models, the mean percentages of reduction in blood transferred was $86 \%$ when a single sterile latex glove was used.
The authors note that their observation that glove material may decrease the exposure volume by $50 \%$ during simulated needlesticks suggests that the risk of infection with human immunodeficiency virus (HIV) or other bloodborne pathogens could be reduced when needles pass through gloves before contacting skin. The magnitude of protection associated with gloves could not be estimated because a linear relationship between transfer volume and infectivity cannot be assumed, especially when the virus titer is high.

The finding that needle size, penetration depth, and glove use affect blood transfer suggests that the accuracy of transmission risk estimates for individual exposures could be improved by accounting for these variables. The authors suggest that postexposure evaluation of all needlestick injuries should document the needle type and size, penetration depth, presence or absence of frank fluid injection, and glove use. In addition, the clinical stage of viral infection and antiviral treatment history of the source patient also should be noted so the magnitude of viremia can be estimated. These data may help clinicians who treat exposed workers and recommend postexposure treatment regimens, such as zidovudine chemoprophylaxis, differentiate between high- and low-risk exposures. The authors conclude that inclusion of these data in surveillance of occupational infection eventually may allow a more refined estimate of magnitude of risk applicable to individual exposure events.

FROM: Mast ST, et al. Efficacy of gloves in reducing blood volumes transferred during simulated needlestick injury. J Infect Dis 1993;168:1589-1592.

\section{Computer-Assisted Education Program for TB}

A new computer-assisted education program, developed by the Association for Professionals in Infection Control and Epidemiology (APIC) and HealthSoft Inc, can be used to assist healthcare facilities meet new federal TB education requirements. This interactive program also includes a posttest and recordkeeping of workers using the program, and can be modified to include site-specific information. For a free preview of "Tuberculosis: Identification, Prevention and Control," call (800) 235-0882.

\section{First Asian-Pacific Conference on Hospital Infection}

The first Asian-Pacific International Conference 